\title{
A NOVEL MECHANISM FOR GENERATING AUXETIC BEHAVIOUR IN RETICULATED FOAMS: MISSING RIB FOAM MODEL
}

\author{
C. W. SMITH*, J. N. GRIMA and K. E. EVANS \\ School of Engineering and Computer Science, Harrison Building, University of Exeter, Exeter, Devon, \\ EX4 4QF, UK
}

( Received 7 February 2000; received in revised form 5 April 2000; accepted 22 May 2000 )

\begin{abstract}
Foams have previously been fabricated with a negative Poisson's ratio (termed auxetic foams). A novel model is proposed to explain this and to describe the strain-dependent Poisson's function behaviour of honeycomb and foam materials. The model is two-dimensional and is based upon the observation of broken cell ribs in foams processed via the compression and heating technique usually employed to convert conventional foams to auxetic behaviour. The model has two forms: the "intact" form is a network of ribs with biaxial symmetry, and the "auxetic" form is a similar network but with a proportion of cell ribs removed. The model output is compared with that of an existing two-dimensional model and experimental data, and is found to be superior in predicting the Poisson's function and marginally better at predicting the stress-strain behaviour of the experimental data than the existing model, using realistic values for geometric parameters. () 2000 Acta Metallurgica Inc. Published by Elsevier Science Ltd. All rights reserved.
\end{abstract}

Keywords: Foams; Elastic; Theory \& modeling; Mesostructure; Poisson’s ratio

\section{INTRODUCTION}

Foam materials with negative Poisson's ratios were first produced by Lakes [1] and have since been examined by several others [2-6]. Lakes described a process by which conventional, reticulated, positive Poisson's ratio foams could be converted into auxetic, i.e., negative Poisson's ratio foams. The process involves volumetrically compressing the foams, heating beyond the polymer's softening temperature and cooling whilst remaining under compression $[1,7,8]$. This process buckles the cell ribs inwards, creating a re-entrant cellular geometry. It is thought that, under tension, the cells unfold outwards towards their original shape, generating an expansion of the bulk specimen and thus a negative Poisson's ratio. This unfolding process reverses under compression. This unfolding is shown for an idealised two-dimensional cell in Fig. 1(a) and (b). The cells in a real foam do not have the symmetry shown in Fig. 1(a) and (b) and the re-entrancy mechanism does not require symmetry to produce a negative Poisson's ratio. However, in order to obtain a closed-form solution for a deter-

* To whom all correspondence should be addressed. Tel.: +44 1392 263652/3615; fax: +44 1392217965 .

E-mail address: c.w.smith@ex.ac.uk (C.W. Smith) (a)

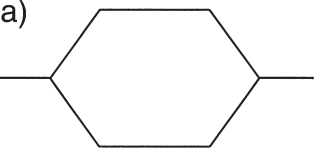

(c)

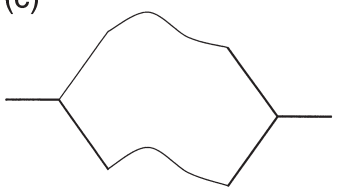

(b)

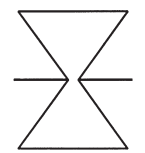

(d)

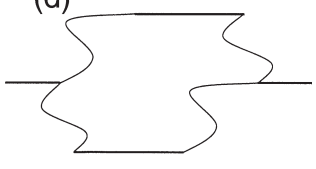

Fig. 1. (a) and (b) The idealised geometry of the re-entrant cell which exhibits auxetic behaviour; (c) and (d) other possible non-symmetrical geometries which do not exhibit auxetic behaviour.

minate system we have chosen to assume symmetry, as shown.

Models have been developed to predict the elastic properties of conventional and auxetic varieties of these materials [9-15]. The models are each based on a particular cellular geometry. Masters and Evans [13] allowed the cell ribs to deform in one of three ways: hinging about their corners, flexing along their lengths, or stretching in tension. Further development of similar cellular geometry based models allowed 
concurrent deformation mechanisms [16]. We have found some of these models to describe the straindependent behaviour of the Poisson's ratio of the conventional foams satisfactorily. They describe the behaviour of the auxetic varieties less well, at least the compression/heat-treated auxetic polyurethane foams produced in our laboratory.

Importantly, we have noted three phenomena in our compression/heat-treated auxetic polyurethane foams. Firstly, as part of the compression and heating process used in our laboratory for conversion of conventional foams to auxetic foams, the foams are twice temporarily removed from the oven and their containers. Once removed they are longitudinally stretched by hand and quickly returned to the containers and oven. This stretching episode, typically repeated twice, is thought to ensure that the cell ribs do not stick to each other. Upon closer inspection we have found that many cells, at least those on the surface, of the auxetic foams have some broken ribs, see Fig. 2. It may be possible that the stretching episodes break some cell ribs. Secondly, the surfaces of the auxetic foams whilst under strain often deform irregularly; some areas clearly deforming more than others. Inevitably, during the volumetric compression of the foams, creases or wrinkles appear in the surface of the foam. It seemed to us that the location of these creases or wrinkles corresponded to the more deformable areas noted following conversion to auxetic behaviour. Thirdly, there is a clear distribution of cell sizes in the foam as supplied, the larger cells being two or more times the size of the smallest, see Fig. 3. We have observed that in the conventional foam the smaller cells deform much less than the larger cells whilst a specimen is under strain.

A novel two-dimensional model is presented that demonstrates a route for conversion of a two-dimensional honeycomb structure from conventional, positive Poisson's ratio to auxetic, negative Poisson's ratio behaviour. The model is then applied to conventional and auxetic varieties of a polyurethane reticu-

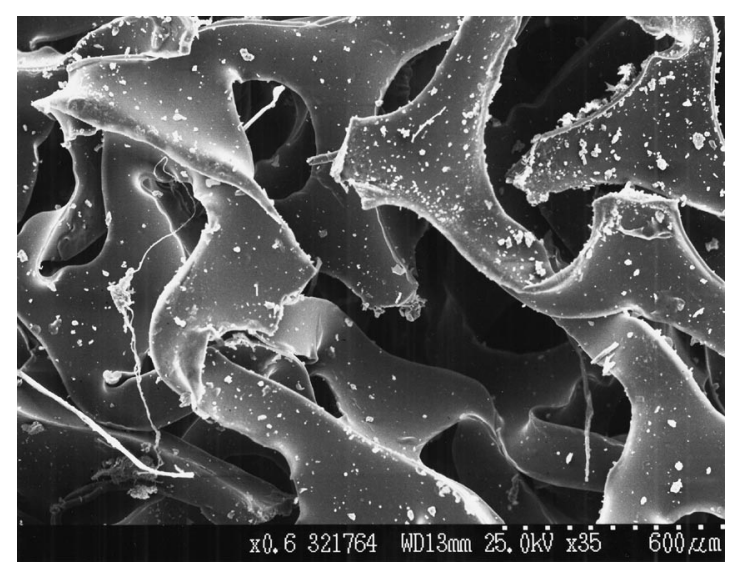

Fig. 2. A cut surface of a foam converted to auxetic behaviour. Note that there are cells with broken ribs lying in the plane of the cut surface.

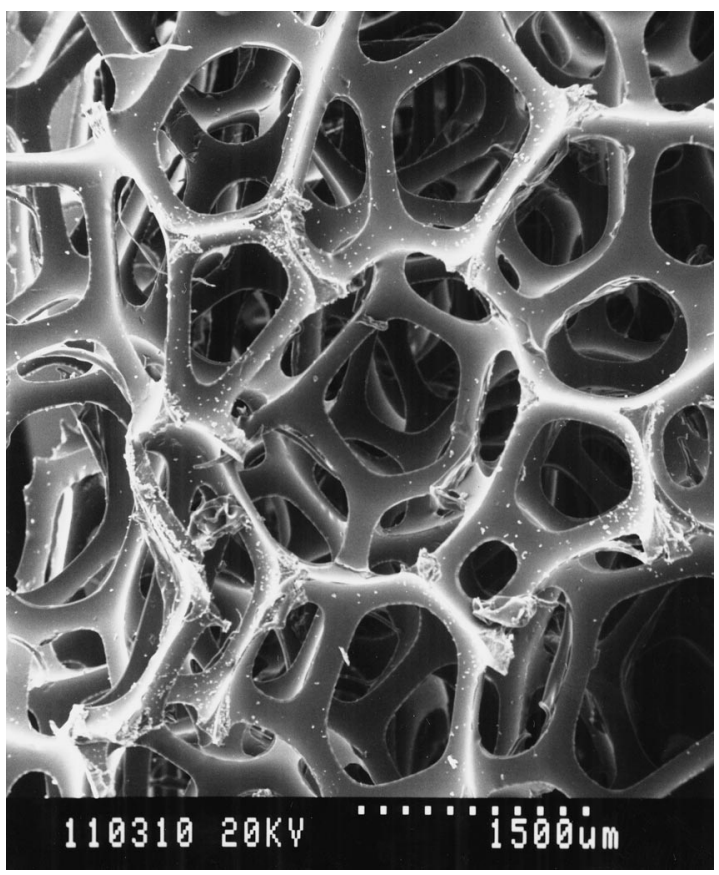

Fig. 3. A cut surface of the foam as supplied. Note cells of different sizes can be seen on the surface.

lated foam, to predict Poisson's ratios and stressstrain behaviour. The new model is similar to previous models in that it is based upon a unit cell that can be tessellated into a network. It is different in that the internal angles are not altered between the conventional and auxetic varieties, instead a fraction of cell ribs are removed. The model is used to predict the strain-dependent Poisson's function rather than the small-strain Poisson's ratio, following the definitions of Smith et al. [17]. The major difference between the two is that the Poisson's function is based upon instantaneous strains and thus better reflects non-linear behaviour than the Poisson's ratio. The instantaneous strain is simply the ratio of the rate of change of length with length at that length, as opposed to the original length as with engineering strain.

\section{EXPERIMENTAL METHODS}

The foam used was reticulated 30 ppi polyester polyurethane ("Filtren" by Recticel Ltd). The gas bubbles that form during synthesis of the foam rise a little, consequently the cells tend to be slightly extended in the vertical direction. Specimens were cut with their long axis in this direction. Specimens were cut with a smooth-edged bandsaw, set up for cutting foam.

The "conventional" specimens were cut as a square section $25 \mathrm{~mm}$ by $25 \mathrm{~mm}$ by $75 \mathrm{~mm}$ long from the foam as supplied. The "auxetic" specimen was cut as a square section $35 \mathrm{~mm}$ by $35 \mathrm{~mm}$ and $105 \mathrm{~mm}$ long and pressed into a mould of $25 \mathrm{~mm}$ by $25 \mathrm{~mm}$ by 75 
$\mathrm{mm}(28.6 \%$ strain along each axis). It was heated at $200^{\circ} \mathrm{C}$ for $10 \mathrm{~min}$ in an oven, then removed from the oven and mould, stretched out by hand for a few moments, and then replaced in the mould and oven for a further $10 \mathrm{~min}$. The removal and stretching by hand was repeated once more. The specimen was then allowed to cool to room temperature whilst remaining in the mould and then heated to $100^{\circ} \mathrm{C}$ for an hour. This technique is adapted from that of Chan and Evans [8], and has been found to be necessary for auxetic behaviour. Foam, as supplied and in the auxetic form, was observed under a stereo microscope and photographs taken.

Both the "conventional" and the "auxetic" specimens were $25 \mathrm{~mm}$ square section and $75 \mathrm{~mm}$ long. End tabs were glued to the specimen ends to aid in gripping in the test machine. The specimens were tested in tension in a universal testing machine (Shimadzu AGS-10kN D). The crosshead was driven for $5 \mathrm{~mm}$ at a rate of $5 \mathrm{~mm} / \mathrm{min}$ and then returned at the same rate for several cycles. All specimens underwent viscoelastic recoverable behaviour.

Strains were measured by a video extensometer system (Videoextensometer, Messphysik $\mathrm{GmbH}$, Austria). The software uses the light intensity information along designated axial and transversal rows of pixels to measure distances between contrasting markers or edges. A contrasting background can be placed behind specimens but axial measurement of displacement requires contrasting markers to be mounted on a specimen's surface. We cut thin strips of white adhesive labelling paper to serve as markers and mounted them approximately one quarter of the specimen's length in from the ends, leaving half the length between them. The software differentiates the light intensity data in order to isolate the sharp transitions between light and dark. The software then records the distance between markers and between edges.

The data output from the video extensometer software-i.e., load (N), distance between axial markers and transverse edges $(\mathrm{mm})$, and sample time (ms) — was imported into a spreadsheet package. All data, including the longitudinal and transversal distance data, inevitably contain some experimental noise. When calculating fractions such as strains and Poisson's ratio, this experimental noise can lead to large errors $[15,16]$. The methods outlined in Smith et al. [17] were used to overcome such problems by smoothing the displacement data with fitted polynomials. Strains and Poisson's ratios were calculated using these smoothed displacement data, and stresses were calculated using the unsmoothed load data and cross-sectional area.

\section{THEORY}

\subsection{Traditional hexagonal re-entrant model}

The traditional two-dimensional model used to describe the behaviour of conventional and auxetic honeycombs and foams is that of Gibson and Ashby [14]. The Poisson's function and Young's modulus in the loading direction are given by

$$
v_{12}=\frac{\sin \theta(h / l+\sin \theta)}{\cos ^{2} \theta}
$$

and

$$
E_{1}=\kappa \frac{(h / l+\sin \theta)}{b \cos ^{3} \theta},
$$

where $h, l, b, \theta$ are as defined in Fig. 4 and $\kappa=$ $E_{\mathrm{s}} b(t / l)^{3}, E_{\mathrm{s}}$ being the intrinsic Young's modulus (the cell is also shown following a small hinging deformation). Experimental evidence suggests that flexure is the dominant deformation mechanism in auxetic foams and in conventional foams with relatively thick ribs such as the foams used in the present study [3, 12]. This honeycomb model was further developed by Masters and Evans [13] for hexagonal cell honeycombs, which may deform concurrently by hinging, flexure and stretching of ribs. In particular, they showed that the expressions for pure hinging, pure flexure and concurrent flexure and hinging are similar to each other and to those of Gibson and Ashby [14].

\subsection{Novel missing rib model}

The observations of the differing cell sizes and the broken ribs formed the conceptual basis for the model. Figure 5(a) shows two idealised networks of cells: an "intact" version and a "cut" version with some ribs removed or broken. The ribs have rotational stiffnesses at their bases, which could arise either from the presence of a small cell or because of a thickened rib base. The large shaded squares cover the areas taken as unit cells, which are shown more clearly in Fig. 5(b).

3.2.1. Intact version. The cells deform by hinging at the base of ribs, and the ribs do not flex or stretch. The cells with four intact ribs are many times stiffer than those with two intact ribs. Thus the intact unit cell deforms solely through a change in the $\zeta$
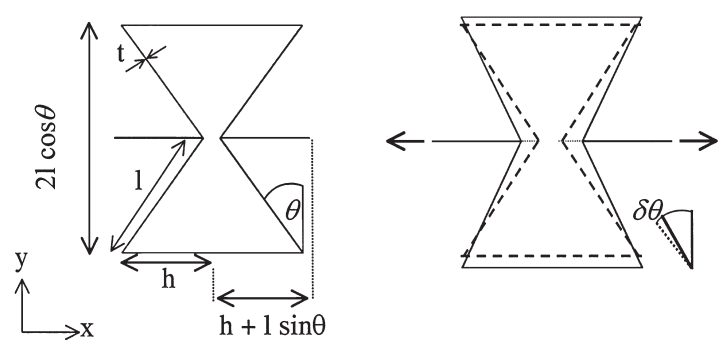

Fig. 4. The hexagonal unit cell of Masters and Evans [13] used to describe some honeycomb and foam materials. The cells ribs have depth $b$ which is not shown here. 

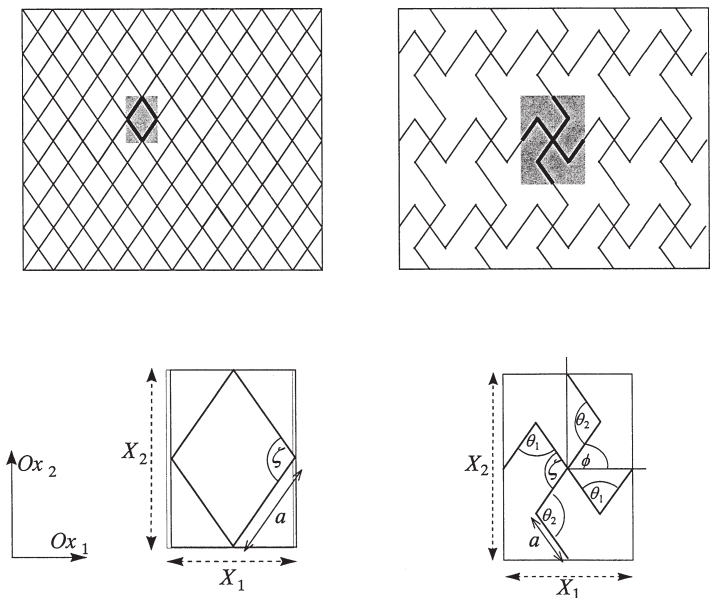

Fig. 5. (a) The idealised networks of the intact version and the cut version with unit cells shaded; (b) the unit cells chosen for the intact and cut versions along with their geometrical parameters.

angle, which has rotational stiffness of $k_{\zeta}$. The intact unit cell has dimensions in the $O x_{i}$ directions given by $(0<\zeta<\pi)$ :

$$
X_{i}=2 a \cos \left(\frac{\zeta}{2}\right)
$$

and

$$
X_{2}=2 a \sin \left(\frac{\zeta}{2}\right)
$$

and hence the Poisson's function is given by:

$$
v_{21}=\left(v_{12}\right)^{-1}=-\frac{\mathrm{d} \varepsilon_{1}}{\mathrm{~d} \varepsilon_{2}},
$$

where $\mathrm{d} \varepsilon_{i}$ is a small incremental strain in the $0 x_{i}$ direction, which may be expressed as:

$$
\mathrm{d} \varepsilon_{i}=\frac{\mathrm{d} X_{i}}{X_{i}}=\frac{1}{X_{i}} \frac{\mathrm{d} X_{i}}{\mathrm{~d} \zeta} \mathrm{d} \zeta
$$

Thus,

$$
\begin{gathered}
v_{21}=\left(v_{12}\right)^{-1}=-\frac{\mathrm{d} \varepsilon_{1}}{\mathrm{~d} \varepsilon_{2}}= \\
-\left[\frac{1}{X_{1}} \frac{\mathrm{d} X_{1}}{\mathrm{~d} \zeta} \mathrm{d} \zeta\right]\left[\frac{1}{X_{2}} \frac{\mathrm{d} X_{2}}{\mathrm{~d} \zeta} \mathrm{d} \zeta\right]= \\
-\frac{X_{2}}{X_{1}} \frac{\mathrm{d} X_{1}}{\mathrm{~d} \zeta}\left[\frac{\mathrm{d} X_{2}}{\mathrm{~d} \zeta}\right]^{-1}
\end{gathered}
$$

where:

$$
\frac{\mathrm{d} X_{1}}{\mathrm{~d} \zeta}=-a \sin \left(\frac{\zeta}{2}\right)
$$

and

$$
\frac{\mathrm{d} X_{2}}{\mathrm{~d} \zeta}=a \cos \left(\frac{\zeta}{2}\right) .
$$

That is,

$$
\begin{aligned}
& v_{21}=\left(v_{12}\right)^{-1}=\frac{2 a \sin (\zeta / 2)}{2 a \cos (\zeta / 2)} \\
& {\left[-a \sin \left(\frac{\zeta}{2}\right)\right]\left[a \sin \left(\frac{\zeta}{2}\right)\right]^{-1}=\tan ^{2}\left(\frac{\zeta}{2}\right) .}
\end{aligned}
$$

The Young's moduli may be derived through the principle of conservation of energy. In a continuum the strain energy per unit volume due to a strain $\mathrm{d} \varepsilon_{i}$ is given by:

$$
U=\frac{1}{2} E_{i}\left(\mathrm{~d} \varepsilon_{i}\right)^{2}
$$

whilst the work done per unit cell by the hinges is equal to:

$$
W=N\left[\frac{1}{2} k_{\zeta}(\mathrm{d} \zeta)^{2}\right],
$$

where $N$ is the number of " $\zeta$ " spring hinges per unit cell, which is equal to 1 [see Fig. 5(b)]. Thus, from the conservation of energy, equations (11) and (12) are related through:

$$
U=\frac{1}{V} W
$$

where $V$ is the volume of the unit cell which, assuming a unit depth in the third axis, is given by:

$$
V=X_{1} X_{2}
$$

Thus combining equations (6) and (11) with equation (14) we obtain:

$$
\begin{aligned}
\frac{1}{2} E_{i}\left(\mathrm{~d} \varepsilon_{i}\right)^{2} & =\frac{1}{2} E_{i}\left(\frac{1}{X_{i}} \frac{\mathrm{d} X_{i}}{\mathrm{~d} \zeta} \mathrm{d} \zeta\right)^{2} \\
& =\frac{1}{X_{i} X_{j}} N\left[\frac{1}{2} k_{\zeta}(\mathrm{d} \zeta)^{2}\right] .
\end{aligned}
$$


and

That is,

$$
E_{i}=N k_{\zeta} \frac{X_{i}}{X_{j}}\left(\frac{\mathrm{d} X_{i}}{\mathrm{~d} \zeta}\right)^{-2},
$$

i.e.,

$$
\begin{array}{r}
E_{1}=4 k_{\zeta} \frac{2 a \cos (\zeta / 2)}{2 a \sin (\zeta / 2)}\left[-a \sin \left(\frac{\zeta}{2}\right)\right]^{-2} \\
=k_{\zeta} \frac{4}{a^{2}} \cot \left(\frac{\zeta}{2}\right) \operatorname{cosec}^{2}\left(\frac{\zeta}{2}\right)
\end{array}
$$

and

$$
\begin{gathered}
E_{2}=4 k_{\zeta} \frac{2 a \sin (\zeta / 2)}{2 a \cos (\zeta / 2)}\left[a \cos \left(\frac{\zeta}{2}\right)\right]^{-2} \\
=k_{\zeta} \frac{4}{a^{2}} \tan \left(\frac{\zeta}{2}\right) \sec ^{2}\left(\frac{\zeta}{2}\right) .
\end{gathered}
$$

3.2.2. Cut version. In the cut version unit cell the joints with angle $\theta$ are spring hinges with spring constants $k_{\theta}$ which are such that $k \zeta \gg k_{\theta}$. Thus we may assume that when the network is loaded in the $0 x_{i}$ direction it deforms solely through changes in the $\theta$ angles. We shall also assume that upon loading in the $O x_{i}$ directions the unit cell remains rectangular.

We may define the unit cell dimensions in the $0 x_{i}$ directions in terms of the single variable $\phi$ by:

$$
X_{1}=4 a \cos (\zeta-\phi)
$$

and

$$
X_{2}=4 a \sin (\phi)
$$

In analogy to equation (8) we have:

$$
\begin{gathered}
v_{21}=\left(v_{12}\right)^{-1}=-\frac{\mathrm{d} \varepsilon_{1}}{\mathrm{~d} \varepsilon_{2}}= \\
-\left[\frac{1}{X_{1}} \frac{\mathrm{d} X_{1}}{\mathrm{~d} \phi} \mathrm{d} \phi\right]\left[\frac{1}{X_{2}} \frac{\mathrm{d} X_{2}}{\mathrm{~d} \phi} \mathrm{d} \phi\right]= \\
-\frac{X_{2}}{X_{1}} \frac{\mathrm{d} X_{1}}{\mathrm{~d} \phi}\left[\frac{\mathrm{d} X_{2}}{\mathrm{~d} \phi}\right]^{-1},
\end{gathered}
$$

where:

$$
\frac{\mathrm{d} X_{1}}{\mathrm{~d} \phi}=4 a \sin (\zeta-\phi)
$$

$$
\frac{\mathrm{d} X_{2}}{\mathrm{~d} \phi}=4 a \cos (\phi)
$$

Thus,

$$
\begin{gathered}
v_{21}=\left(v_{12}\right)^{-1}=-\frac{4 a \sin (\phi)}{4 a \cos (\zeta-\phi)} 4 a \sin (\zeta \\
-\phi)[4 a \cos (\phi)]^{-1}=-\tan (\phi) \tan (\zeta-\phi) .
\end{gathered}
$$

The Young's moduli for this network may also be derived through a conservation of energy approach. In particular, the strain energy per unit volume $U$ and the work done per unit cell $W$ are given by:

$$
U=\frac{1}{2} E_{i}\left(\mathrm{~d} \varepsilon_{i}\right)^{2}=\frac{1}{2} E_{i}\left(\frac{1}{X_{i}} \frac{\mathrm{d} X_{i}}{\mathrm{~d} \phi} \mathrm{d} \phi\right)^{2}
$$

and

$$
W=N_{1}\left[\frac{1}{2} k_{\theta}\left(\mathrm{d} \theta_{i}\right)^{2}\right]+N_{2}\left[\frac{1}{2} k_{\theta}\left(\mathrm{d} \theta_{2}\right)^{2}\right],
$$

where $N_{1}$ and $N_{2}$ are the number of " $\theta_{1}$ " and " $\theta_{2}$ " hinges, respectively; i.e., $N_{1}=N_{2}=2$. Furthermore, from simple geometry, the terms " $\mathrm{d} \theta_{1}$ " and " $\mathrm{d} \theta_{2}$ " may be re-expressed in terms of " $\mathrm{d} \phi$ " by:

$$
\mathrm{d} \theta_{1}=\mathrm{d} \theta_{2}=2 \mathrm{~d} \phi \text {. }
$$

Hence equations (13) and (14) together with equation (27) may be combined to give:

$$
\begin{array}{r}
\frac{1}{2} E_{i}\left(\frac{1}{X_{i}} \frac{\mathrm{d} X_{i}}{\mathrm{~d} \phi} \mathrm{d} \phi\right)^{2}=\frac{1}{X_{1} X_{2}}\left\{2\left[\frac{1}{2} k_{\theta}\left(\mathrm{d} \theta_{1}\right)^{2}\right]\right. \\
\left.+2\left[\frac{1}{2} k_{\theta}\left(\mathrm{d} \theta_{2}\right)^{2}\right]\right\}=\frac{2 k_{\theta}(\mathrm{d} \phi)^{2}}{X_{1} X_{2}},
\end{array}
$$

which simplifies to:

$$
E_{i}=4 k_{\theta} \frac{X_{i}}{X_{j}}\left(\frac{\mathrm{d} X_{i}}{\mathrm{~d} \phi}\right)^{-2} .
$$

Thus,

$$
\begin{gathered}
E_{1}=4 k_{\theta} \frac{4 a \cos (\zeta-\phi)}{4 a \sin (\phi)}[4 a \sin (\zeta-\phi)]^{-2} \\
=k_{\theta} \frac{1}{4 a^{2}} \frac{\cot (\zeta-\phi)}{\sin (\phi) \sin (\zeta-\phi)}
\end{gathered}
$$


and

$$
\begin{array}{r}
E_{2}=4 k_{\theta} \frac{4 a \sin (\phi)}{4 a \cos (\zeta-\phi)}[4 a \cos (\phi)]^{-2} \\
=k_{\theta} \frac{1}{4 a^{2}} \frac{\tan (\phi)}{\cos (\phi) \cos (\zeta-\phi)} .
\end{array}
$$

Note that the derived Poisson's functions and the Young's moduli satisfy the symmetry requirement:

$$
\frac{v_{12}}{E_{1}}=\frac{v_{21}}{E_{2}} .
$$

\section{RESULTS}

The differing cell sizes of the foam, as supplied, can be seen in Fig. 3(a). Broken ribs have been highlighted in Fig. 3(b), which shows the surface of the auxetic form of that foam. Some broken ribs in the auxetic foam lie in a plane parallel with that of the cut surface, distinct from the conventional foam which has no such broken ribs. The absence of broken ribs in the plane of the cut surface of the conventional foam suggests that the ribs were not broken during the cutting process.

The length and width data of the foam specimens in tension are shown in Fig. 6. The data show that the conventional specimen got thinner as it was stretched whilst the auxetic specimen got wider. Figure 7 shows fits of the broken rib model and the hexagonal model to both types of foam, auxetic and conventional. The model data shown was the best fit possible with the experimental data. The value of $\theta$ in the hexagonal model was negative when fitted to the conventional foam and positive when fitted to the auxetic foam. Selected ribs were removed from the unit cell to fit the missing rib model to the auxetic foam and left in situ to fit the conventional foam. The parameter

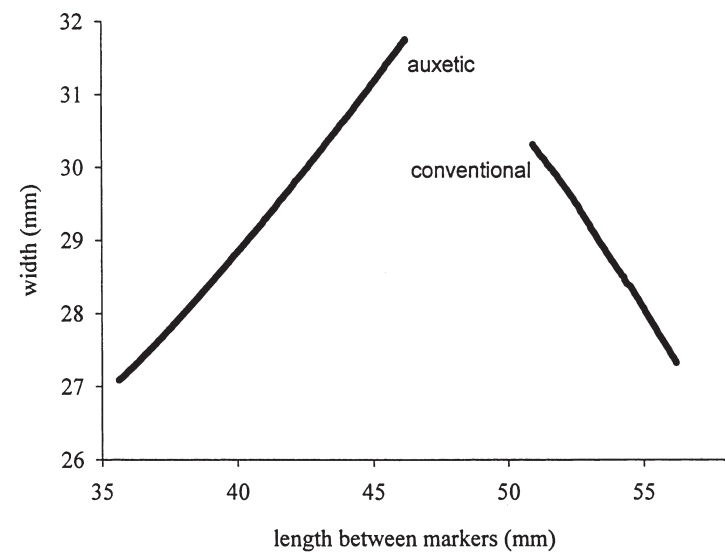

Fig. 6. Length and width data of the conventional and auxetic foam specimens. The conventional specimen decreases in width whereas the auxetic specimen increases in width under tension.

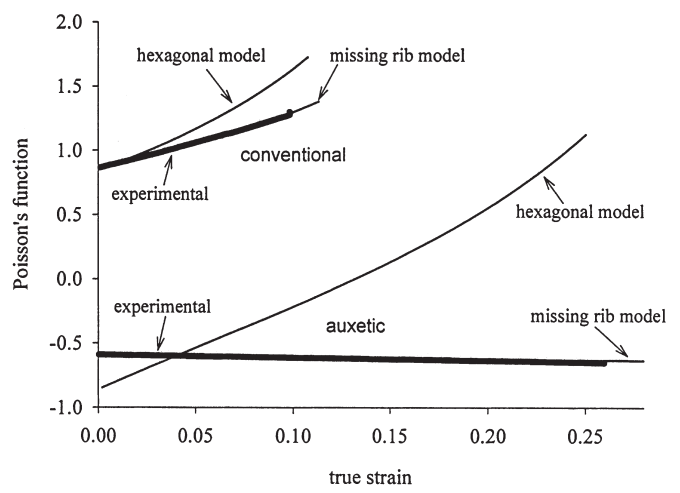

Fig. 7. Poisson's function and true strain data of the foam specimens, both versions of the missing rib model, and both versions of the hexagonal model. Experimental data are shown as thick lines and model predictions as thin lines, and all are labelled.

Table 1. The values of the parameters used to calculate the Poisson's function data in Fig. 7 for the hexagonal and missing rib models

\begin{tabular}{lcc}
\hline Hexagonal model & Re-entrant & Conventional \\
\hline$h$ & 2 & 2 \\
$l$ & 1 & 1 \\
$\theta\left({ }^{\circ}\right)$ & -25.99 & 19.2 \\
$\kappa$ & 0.03 & 0.03 \\
\hline
\end{tabular}

\begin{tabular}{lcc}
\hline Missing rib model & Cut version & Intact \\
\hline$a$ & 1 & 1 \\
$\zeta\left(^{\circ}\right)$ & 77.3 & 85.7 \\
$\phi\left({ }^{\circ}\right)$ & 24.1 & - \\
$\theta_{1}$ and $\theta_{2}\left({ }^{\circ}\right)$ & 73.4 and 48.1 & - \\
$K_{\theta}$ and $K_{\zeta}$ & $K_{\theta}=0.08333$ & $K_{\zeta}=0.01429$ \\
\hline
\end{tabular}

values used for both models in Fig. 7 are given in Table 1.

The true stress-true strain behaviour of the experimental specimens, and best-fitting predictions of both versions of the missing rib and hexagonal model, are shown in Fig. 8. The conventional foam is stiffer than

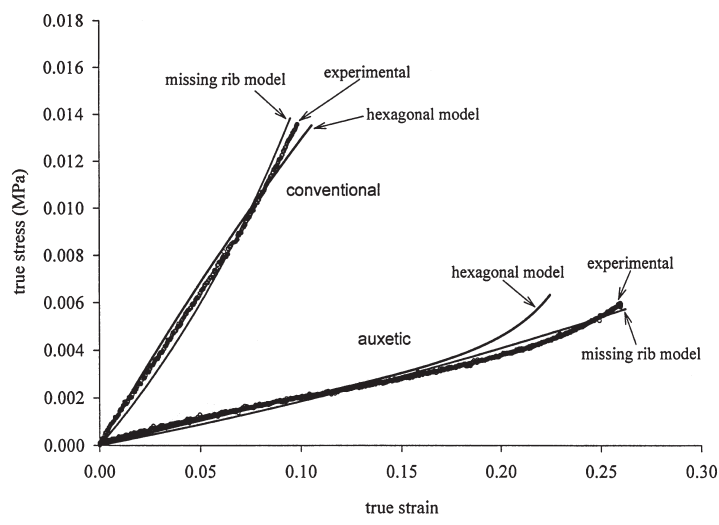

Fig. 8. True stress and true strain data of the conventional and auxetic specimens, and similar data from both versions of the missing rib and hexagonal models. The experimental data are drawn as circles and the model data as solid lines. 
the auxetic specimen, which is typical of this particular foam material [3, 18]. Both the hexagonal and missing rib models predict a higher value of $E$ for the conventional foam than the auxetic. The models predict the true stress-true strain behaviour reasonably well, especially considering the error likely in the experimental data. The values of the geometrical parameters $\zeta, \phi$ and $a$ used in these calculations are similar to those used in the calculations for the bestfitting Poisson's function in Fig. 7.

The geometric parameters of the intact version of the new model were set so that the unit cell was square, i.e., $\zeta=\pi / 2\left(90^{\circ}\right)$ and $\phi=\pi / 4\left(45^{\circ}\right)$; thus the unit cell for the cut version differed only by removal of selected ribs. The predictions of the two versions with these parameters are shown as solid lines in Fig. 9. In an effort to reflect the anisotropy exhibited in many foams due to the rising of gas bubbles during manufacture, we set the angle $\zeta=2 \pi / 3\left(120^{\circ}\right)$ and $\phi=50^{\circ}$ (the maximum angle without overlapping of adjacent unit cells) in the two versions of the model. The model predictions with these parameter values are shown as dashed lines in Fig. 9.

\section{DISCUSSION}

The method of heating whilst under compression has been shown to be successful in converting conventional, positive Poisson's ratio foams into auxetic, negative Poisson's ratio foams [1]. Our evidence suggests that this process may also break some cell ribs in the polyurethane foam described.

It is evident that the traditional two-dimensional hexagonal honeycomb model of Masters and Evans [13] presently used to describe the behaviour of foams such as those studied here, is sufficient for the conventional variety but is inaccurate in reproducing the strain-dependent behaviour of the Poisson's function/ratio of the auxetic varieties. The new missing rib model can be made to describe the behaviour of both varieties using realistic cell geometries. This demonstrates an alternative possible route to conver-

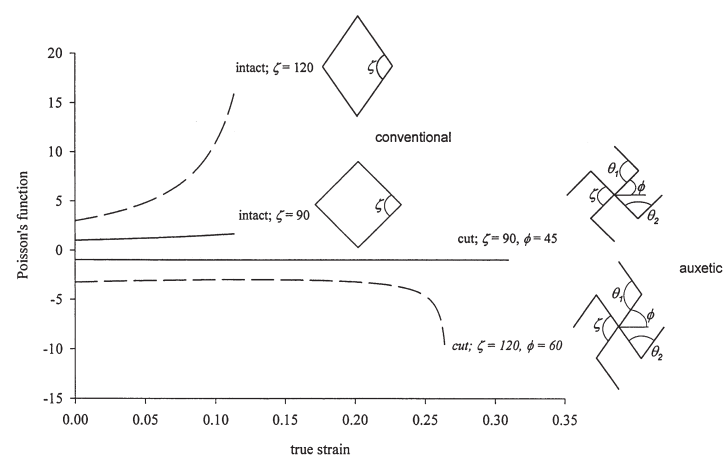

Fig. 9. Poisson's function and true strain data predictions of both versions of the missing rib model; square unit cell (solid line) and elongated unit cell (dashed line). The geometric parameters are $\zeta=\pi / 2$ and $\phi=\pi / 4$, and $\zeta=2 \pi / 3$ and $\phi=\pi / 3$, respectively, for the square and elongated unit cells (shown in ${ }^{\circ}$ ). sion to auxetic behaviour. The new missing rib model is also slightly better at predicting the stress-strain behaviour of the present foam type. This may suggest that, in some cases, in compression and heat-treated foams there may be more than one mechanism operating to produce auxetic behaviour: i.e., concurrent out-folding of re-entrant cells and the missing ribs rotation mechanism. Direct evidence of this should be sought and may be possible via confocal microscopy methods.

The selective removal of ribs in the missing rib model could be criticised as being unrealistic, as could the symmetry of the hexagonal model. The hexagonal model probably does not require symmetry for auxetic behaviour but it was assumed so that the unit cell was determinate and simple expressions could be derived. It could be expected that in a real foam there would be a distribution of broken ribs within cells and the distribution of re-entrant and broken rib cells would be random to a degree. It is also likely that real foams will contain both re-entrant and missing rib cells. The model was not intended to accurately reflect the conditions in the compression/heat-treated foam, rather to explore a new mechanism. There seems to be no existing method at present for modelling the behaviour of non-regular cells and non-periodic networks of cells, except explicit numerical approaches [19, 20]. The effects of a network with only a proportion of unit cells with missing ribs, and that of a more random distribution of missing ribs, might be fruitfully explored using methods similar to those of Fortes and Ashby [21].

The best-fitting match to the experimental data for the missing rib model had values of the stiffness constants $k_{\theta}>k_{\zeta}$ contrary to the assumption in the theory that $k_{\zeta} \gg k_{\theta}$, see Table 1. This is not inconsistent as the two experimental foam specimens were not similar; the auxetic specimens have a higher density, typically by a factor of two or three, and auxetic specimens undergo compression/heat treatment. The auxetic specimen will therefore have a higher density of load-bearing hinges per unit volume than the conventional specimen, and the polyurethane polymer itself may have become stiffer due to its treatment.

When the geometry of the cell is set to a square the intact version has an initial Poisson's function value of 1 and the cut version has a value of -1 . The unit cell of the cut version undergoes concurrent outfolding and rotation which keeps the Poisson's function/ratio value constant, whereas the value of the intact version increases due to its "scissors" deformation mechanism.

Further development of this model would include extending it into the third dimension and exploring its off-axis properties. The model suggests a possible new route for the manufacture of auxetic foam type materials, bypassing the need for compression and heating stages. This removal could be accomplished by a variety of chemical or physical means, with control over the final properties. Such a technique could 
be more practical for large volumes of foam than the current heating/compression method.

\section{CONCLUSION}

We have developed a novel model to describe auxetic behaviour in two dimensions of a honeycomb or foam type material, termed the missing rib model. It is based upon the selective removal of ribs from a network without changes of internal angles. The missing rib model is better at predicting the strain-dependent Poisson's function/ratio and stress-strain behaviour of the reticulated auxetic polyurethane foam we tested. It suggests a possible new route for the direct manufacture of auxetic foams.

Acknowledgements-C. W. Smith was supported by the BBSRC of the UK during the period of this work. J. N. Grima was supported during this work by the University of Exeter and the CVCP of the UK.

\section{REFERENCES}

1. Lakes, R. S. Science, 1987, 235, 1038

2. Lakes, R. S. and Elms, K. J. J. Compos. Mater., 1993, 27, 1193.

3. Chan, N. and Evans, K. E. J. Mater., 1997, 32(21), 5725.
4. Lee, T. and Lakes, R. S. J. Mater. Sci., 1997, 9(9), 2397.

5. Chan, N. and Evans, K. E. J. Cellular Plast., 1998, 34, 231.

6. Smith, C. W., Lehman, F., Wootton, R. J. and Evans, K. E. Cellular Polym., 1999, 18(18), 79.

7. Friis, E. A., Lakes, R. S. and Park, J. B. J. Mater. Sci., 1988, 23, 4406.

8. Chan, N. and Evans, K. E. J. Mater. Sci., 1997, 32(22), 5945.

9. Gibson, L. J., Ashby, M. F., Shajer, G. S. and Robertson, C. I. Proc. Roy. Soc. London A, 1982, 382(1782), 25.

10. Evans, K. E. J. Phys. D: Appl. Phys., 1989, 22, 1870.

11. Choi, J. B. and Lakes, R. S. Int. J. Mech. Sci., 1995, 37(1), 51.

12. Choi, J. B. and Lakes, R. S. J. Compos. Mater., 1995 29(1), 113.

13. Masters, I. G. and Evans, K. E. Compos. Struct., 1996, 35, 403.

14. Gibson, L. J. and Ashby, M. F. Cellular Solids: Structure and Properties, 2nd ed. Cambridge University Press, Cambridge, UK, 1997.

15. Zhu, H. X., Knott, J. F. and Mill, N. J. J. Mech. Phys. Solids, 1997, 45(3), 319.

16. Alderson, A. and Evans, K. E. J. Mater. Sci., 1997, 37, 1.

17. Smith, C. W., Wootton, R. J. and Evans, K. E. Exp. Mech., 1999, 39(4), 1.

18. Chan, N. and Evans, K. E. J. Cellular Plast., 1999, 35, 130.

19. Silva, M. J., Hayes, W. C. and Gibson, L. J. Int. J. Mech. Sci., 1995, 37(11), 1161.

20. Silva, M. J. and Gibson, L. J. Int. J. Mech. Sci., 1997, 39(5), 549.

21. Fortes, M. A. and Ashby, M. F. Acta mater., 1999, 47(12), 3469. 